\title{
The feasibility of a multi-professional training to improve how healthcare professionals deliver different news to families during pregnancy and at birth.
}

\begin{abstract}
Background

In the UK, pregnant women are offered fetal anomaly screening to assess the chance of their baby being born with eleven different conditions. How healthcare professionals (HCPs) deliver news about a child having a congenital anomaly affects how it is received and processed by parents. We refer to this news as different news.
\end{abstract}

\section{Methods}

We conducted a mixed methods evaluation of a training intervention to improve how HCPs deliver different news. 26 HCPs self-completed pre and post-training questionnaires on skills, knowledge, and attitudes related to delivering different news. Interviews were conducted with 8 HCPs. Quantitative data were analysed using descriptive statistics, and the paired t-test to compare the pre and post scores, estimate the difference between pre and post scores and the 95\% confidence interval. Qualitative data were analysed using Framework guided by the Theoretical Domains Framework (TDF).

\section{Results}

The training intervention was both feasible and acceptable. HCPs indicated that it enhanced or consolidated their knowledge and skills, covered topics relevant to their practice and that they would recommend it to colleagues. Participants particularly valued integration of the voice of parents with lived experience in the training. Significant increase in mean scores were observed in confidence to deliver different news $(2.81,95 \% \mathrm{Cl} 2.43$ to 3.19; to $4.2895 \% \mathrm{Cl} 4.09$ to 4.47; $\mathrm{P}<0.001)$ and skills to deliver different news $(3.00,95 \% \mathrm{Cl} 2.64$ to 3.36 to $4.36,95 \% \mathrm{Cl} 4.13$ to $4.59 ; \mathrm{P}<0.001)$. HCPs reported feeling more confident in their ability to provide sensitive, responsive, balanced care to families.

\section{Conclusions}

The significant improvements in confidence and skills reported by HCPs suggest that the training may be effective in equipping HCPs to minimise the distress, anxiety, and depression associated with receiving different news. This represents a key aspect of the prevention of mental ill health across the life course. 


\section{Introduction}

In 2016, nearly 1 in 49 babies born in England had a congenital anomaly (NCARDRS, 2018). This includes anomalies associated with a learning disability such as Down syndrome which may be identified as part of the NHS Fetal Anomaly Screening Programme (PHE, 2018). Other congenital anomalies are only diagnosed at birth or when the child begins to miss key developmental milestones and further investigations result in a confirmed diagnosis. The Fetal Anomaly Screening Programme also identifies other conditions which are not associated with learning disability however this study specifically focused on conditions associated with a learning disability in response to a commissioned call for research funding focusing on this subject.

There is evidence to suggest that how healthcare professionals (HCPs) tell parents about their child having a congenital anomaly affects both the way parents adjust to the situation and the wellbeing of their child (RCN, 2013). In this study, we refer to this process as delivering different news (DDN), which is the process of imparting information relating to a child being diagnosed with a condition associated with a learning disability during pregnancy or at birth. Receiving different news has been reported as distressing and unexpected with parents experiencing various emotions such as anger, guilt, sadness and depression (Dent \& Carey, 2006; Luz, George, Spitz, \& Vieux, 2017; Mugweni et al., 2019).

These emotions are of particular importance in the first 1001 days of a child's life (preconception -2 years old) as parent experiences of chronic stress; depression, anxiety or other mental health conditions may affect foetal programming and the parent-foetus relationship - and post birth this may affect the parentinfant relationship (Wen et al., 2017). This, in turn, can impact on the social-emotional, cognitive and physical development of children, increasing the risk of mood disorders in later life (Deater-Deckard, 2005). Maternal and paternal depression are also known predictors of poorer cognitive functioning, impaired adaptive functioning, increased risk of depression, anxiety and conduct disorders in some children exposed to this (Drury, Scaramella, \& Zeanah, 2016; Hoffman, Dunn, \& Njoroge, 2017; Stein et al., 2014).

It is, therefore, crucial to ensure that every parent who receives different news has access to a HCP who is trained to minimise the negative psychological impact of the news and maximise psychological wellbeing through effective support of the whole family. Currently, this is not the case for every family due to lack of standardised training or policy to guide professionals on how to effectively deliver DN (Luz et al., 2017; RCN, 2013).

\section{Methods}

We conducted a two-phase study to assess the feasibility and acceptability of a training intervention to improve how healthcare professionals deliver different news to parents. This paper is based on phase 2 of 
the study. Findings from phase 1 have been described fully elsewhere In brief, intervention development drew on the Theoretical Domains Framework (TDF) version 2 (Cane, O'Connor, \& Michie, 2012) to identify barriers and enablers to efficient delivery of DN using qualitative data from phase 1 . The functions of the intervention required to improve the process of DDN, and the corresponding evidenced based behaviour change techniques were identified through examination of the COM-B model and the Behaviour Change Taxonomy. Viability of the intervention was assessed using APEASE criteria (Acceptability, Practicability, Effectiveness/cost-effectiveness, Affordability, Safety/side-effects, Equity) (Michie, Atkins, \& West, 2014) and the final intervention was specified. We developed a READY framework (figure 1) for effective delivery of different news.

\section{Sampling and data collection}

We received ethics approval from the Cambridge East NRES Research Ethics Committee

All staff who deliver different news in NHS Trusts supported by Health Education England working across Kent, Surrey and Sussex were invited to participate in a half-day training workshop in Crawley and Canterbury. The training was delivered by the research team EM, MW and AEJ. EM presented the study findings from Phase 1. AEJ, our parent representative, described her own journey of receiving different news, what went well and what could have gone better as well as the immediate and long-term impact of the news on herself and the family. MW facilitated a session on sharing and learning from practice and case studies based on actual real-life experiences shared by families during phase 1 of the study. Participants completed pre and post-training questionnaires on skills, knowledge, and attitudes related to DDN on a five-point Likert scale from 1 indicating strongly disagree and 5 strongly agree. The post training questionnaire also sought feedback on the content of the training.

As a feasibility study, the study was not powered to detect statistical significance. We recruited 26 HCPs to the training as per recommendations for pilot and feasibility studies which propose examining changes in a group of 24 to 50 subjects in order to estimate parameters for a follow on randomised control trial (Lancaster, Dodd, \& Williamson, 2004). 8 HCPs registered their interest in qualitative telephone interviews a month after the training. Interviews were conducted by EM. They explored perceived changes in knowledge, attitude, and practices after the training and sought additional feedback on the training. This number allowed for saturation in terms of scope and replicability (Morse, 2015). Interviews were conducted using an interview guide and lasted between 25 and 45 minutes. They were audio-recorded and transcribed. Interviewer bias was reduced by involving the whole team in the development of the interview guide and cross checking data interpretations. 


\section{Data Analysis}

All quantitative analyses were carried out in SPSS using descriptive statistics, and the paired t-test to compare the pre and post scores, estimate the difference between pre and post and $95 \%$ confidence intervals as per recommendations of analysing Likert scale data with five or more categories. This was conducted by EM and SK. All qualitative data were managed using NVivo and analysed by EM, CL, SK, SJ using Framework analysis (Ritchie \& Spencer, 1994) guided by the Theoretical Domains Framework (TDF) version 2 (Atkins et al., 2017; Cane et al., 2012). Framework begins with familiarisation with the data, followed by the development of a thematic framework for indexing the data (Ritchie \& Spencer, 1994). Data is then charted followed by mapping and interpretation which allows the development of descriptive and explanatory accounts of the findings illustrated by anonymised quotations (Ritchie \& Spencer, 1994). Data interpretations were cross-checked across EM, CL, SK, SJ and MW. Member checks were achieved by checking with two of the research participants who reviewed the findings before publication of the research report.

\section{FIGURE 1:}

\section{Results}

\section{Description of Participants}

26 HCPs participated in the Phase 2 training. Their professions are indicated in figure 2 below.

\section{Figure 2}

8 HCPs agreed to participate in semi-structured interviews four weeks after training. Due to this small number, their demographic details are not specified in order to protect their identity.

\section{Feasibility and Acceptability}

Feasibility of the training was assessed by the percentage of eligible participants who eventually enrolled in the training. 37 participants had registered for the training and 26 participants attended (70.5\%). There were various reasons for withdrawal such as illness, family emergencies and some unexplained. Acceptability was assessed by the percentage of HCPs who completed all aspects of the training as planned. All participants (100\%) completed all aspects of the training as planned.

The quantitative and qualitative phase 2 findings are presented under the TDF that were relevant to the study and which emerged from the data. 


\section{Knowledge, skills and beliefs about capabilities}

The training aimed to enable HCPs to understand the impact of DN on parents and recognise the importance of establishing the right physical, emotional, social and environmental space to deliver DN.

We observed significant increase in mean scores relating to : understanding the effect of DN on parents (3.89, $95 \% \mathrm{Cl} 3.55$ to 4.21 ; to $4.89,95 \% \mathrm{Cl} 4.75$ to $5.02 ; \mathrm{P}<0.001$ ); importance of empathy when delivering different news ( $4.04,95 \% \mathrm{Cl} 3.77$ to 4.31 ; to $4.6995 \% \mathrm{Cl} 4.50$ to 4.88 ; $\mathrm{P}<0.001)$; confidence to deliver DN (2.81, $95 \% \mathrm{Cl} 2.43$ to $3.19 ;$ to $4.2895 \% \mathrm{Cl} 4.09$ to $4.47 ; \mathrm{P}<0.001)$ and skills to deliver $\mathrm{DN}(3.00,95 \% \mathrm{Cl} 2.64$ to 3.36 to $4.36,95 \% \mathrm{Cl} 4.13$ to $4.59 ; \mathrm{P}<0.001)$.

These findings were also confirmed in the qualitative data. Interview participants reported feeling more confident in their ability to share DN compassionately. Some regrets were expressed about the way they interacted with parents prior to attending the training:

..that lady's probably going to remember that for the rest of her life, she probably is, but there's nothing I can do about it now, all I can do now is change the language I use. (HCP 8)

Nearly all the participants commented on the value of learning about the importance of all aspects of communication, including the words and tone used and replacing the term 'bad news' with 'different news'. There were some immediate changes in practice reported by participants, for example, changing the standardised wording of follow-up emails sent to parents to reflect the content of the training.

\section{Social / Professional roles and identity and Social influences}

Most interview participants expressed their sense of privilege at being able to deliver DN to families. There was also a clearly stated belief that all HCPs who deliver DN needed appropriate training. Only $30.8 \%(n=8)$ of the training participants indicated that they had received some training in DDN. The majority $57.7 \%$ $(n=15)$ had not received training and data were missing from 3 participants $(11.5 \%)$ as shown in figure 3 :

\section{Figure 3}

Data from open ended questions in the questionnaires as well as the interviews indicated that within the training, the most powerful interpersonal influences that seemed to affect the impact of the training and its subsequent implementation in practice were the parent testimony and case studies. The interactive nature of the training was also mentioned as providing opportunities for attendees to share ideas and experiences and to learn from each other. 


\section{Environmental context and Resources}

Positive comments were made about the venue, the refreshments, the atmosphere, the other attendees, the facilitators, the mother with lived experience, the content of the training and the way it was delivered. This was also reflected in the quantitative data in that $96.2 \%$ of participants $(n=25)$ agreed that the training covered topics relevant to their practice; that the content was well organised and easy to follow; that interaction and discussion were encouraged during the training. Data were missing for one participant. In the qualitative comments, participants valued various aspects of the training:

I think you always get quite a lot back from a service user's point of view, so the lady that spoke about her own case with her child... (HCP 2)

The scenarios where we did go in groups, that was really nice as it wasn't particularly threatening in a sense of you have to answer it all because it was opened up to the whole group, actually I really liked that and hearing different people's opinions was really good. (HCP 3)

\section{Optimism}

The training aimed to help HCPs to understand the importance of providing a balanced description of congenital anomalies in order to empower families to see beyond their child's diagnosis and build positive relationships with them. In the post-training questionnaires, significant changes were observed in the domain of understanding how to provide a balanced description of a condition with a mean score change from $(2.84,95 \% \mathrm{Cl} 2.46$ to 3.24 ; to $4.346,95 \% \mathrm{Cl} 4.07$ to 4.62 ; $\mathrm{t}=6.93, \mathrm{P}<0.001)$.

The resources shared at the training such as the "Looking Up" book, the "Tea at Grandma's Book" and the "Going to school book" were perceived as contributing to HCPs having a balanced understanding of the realities of raising a child with congenital anomalies:

... standard national literature that we're given to use is so old fashioned and so negative...,, it was lovely to see some positive stuff. But also, once you got past the initial positive bit, it had realism there, you know, it wasn't sort of sugar-coated silliness. (HCP 5)

\section{Beliefs about consequences}

In this domain we explored the potential positive impact of training HCPs to deliver DN on the outcomes and experiences of families receiving DN. The HCPs felt the need to ensure safe, consistent balanced practice knowing the long-term impact that their practice would have on parents:

.. that they really remember sort of like the tone and the way in which they were spoken to, so to always go in and give them the respect that this might be one of the few interactions and they'll remember it longer than you ever will. (HCP 7)

All participants $(n=26)$ stated that they would recommend the training to colleagues. From the qualitative interviews, the expected outcomes related not only to their practice but also to the extent to which the training could be rolled out and embedded in practice for all HCPs. There were several suggestions about 
ensuring that the infrastructure required for supporting the establishment of protocols and policies for effectively DDN.

\section{Emotion}

The training aimed to allow HCPs to recognise the importance of taking care of their own emotional and mental wellbeing. There was a unanimous acknowledgment by all the participants of the emotional challenges of supporting families facing uncertain futures, especially when the decisions that some families made were difficult to reconcile with the HCPs own beliefs:

You know, they do play on your mind some of these lady's cases and you're just hoping...in the long run ...in the long run that the baby's forgiving you.... (HCP 1)

The need for a supportive network in order to reduce stress and avoid burn-out was mentioned by several participants, so the session on novel approaches to build practitioner resilience was also considered helpful. There was a significant increase in mean score on being able to manage one's emotions related to delivering $\mathrm{DN}(3.15,95 \% \mathrm{Cl} 2.76$ to 3.54 ; to $4.27,95 \% \mathrm{Cl} 4.09$ to 4.45 ; $\mathrm{P}<0.001)$.

\section{Discussion}

We conducted a two-phase study to develop and assess the feasibility and acceptability of a training intervention to improve the delivery of DN to families by HCPs. Our training did not focus on the decision to continue or terminate a pregnancy based on fetal anomaly screening but aimed to equip healthcare professionals to demonstrate empathy; show compassion; be flexible with time or plan around the demands of their role; utilise kind, simple and truthful language; offer sufficient time to answer questions; know when and where to refer families on to for further care and support These key aspects of DDN have also been highlighted in other studies as crucial for minimising the negative impact of receiving different news on parents (Luz et al., 2017; Sheets, Baty, Vazquez, Carey, \& Hobson, 2012; Skotko \& Bedia, 2005).

We found that it was both feasible and acceptable to deliver the training intervention to multiprofessionals involved in the prenatal and postnatal delivery of different news. We also found that it was both feasible and acceptable to make use of real-life scenarios in the case studies alongside learning from the voice of parents with lived experience during the training. It would be important to ensure that the voice of parents with lived experience remains part of future training opportunities so that it continues to reflect lived experiences and the possible long-term impact on families. This component could be strengthened by including the lived experience of a range of other congenital anomalies and carers as well as incorporating ways to support families that choose to terminate a pregnancy based on foetal anomaly screening results. 
All participants indicated that attendance at the half-day DDN training workshop enhanced or consolidated their knowledge and skills, that it covered topics which were relevant to their current practice and that they would recommend the training to their colleagues. Significant increase in mean scores were observed in : understanding of the effect of DN on parents was reported $(3.89,95 \% \mathrm{Cl} 3.55$ to 4.21 ; to $4.89,95 \% \mathrm{Cl}$ 4.75 to 5.02 ; $\mathrm{P}<0.001)$; confidence to deliver $\mathrm{DN}(2.81,95 \% \mathrm{Cl} 2.43$ to 3.19 ; to $4.2895 \% \mathrm{Cl} 4.09$ to 4.47 ; $\mathrm{P}<0.001)$ and skills to deliver $\mathrm{DN}(3.00,95 \% \mathrm{Cl} 2.64$ to 3.36 to $4.36,95 \% \mathrm{Cl} 4.13$ to $4.59 ; \mathrm{P}<0.001)$. These are important findings which suggest that the training could equip HCPs to reduce the negative psychological impact of receiving DN on parents. On their return to their workplace participants felt better informed and more confident in their ability to provide sensitive, responsive, balanced care when supporting families. Several HCPs reported practical changes which they had already made in their daily practice as a result of the training. Like other authors, we found that the use of the TDF in guiding the development of the intervention was useful in developing training which addressed some of the challenges and barriers to DDN effectively.

The study has a number of limitations. As a feasibility study, this study was not powered to detect statistical significance. It would be important to conduct of a larger definitive large-scale trial as a next step. Other limitations stem from the small sample size in the qualitative aspect which means the results are not generalisable but are transferable. Training invitations were sent to all HCPs who deliver different news but it is possible that only those who felt they needed to improve their skills in this area or have a special interest in this area chose to participate in the training which would have a possible impact on the results of the study. The preliminary results from this study will therefore need to be tested in a much larger study.

This follow on study could look at the implementation of the training and its impact on family outcomes and answer questions about how HCPs who have been trained to deliver DDN might improve the emotional response and mental wellbeing of parents immediately after the news is delivered and in subsequent months. Other studies have shown that parents may take six or more months to adjust to the news and to develop the emotional resilience to enable common family function (Fonseca, Nazare, \& Canavarro, 2014). A cross-sectional survey of parents found that over a period of six months, parents who were well supported and had access to appropriate information when they needed it, adjusted well to the diagnosis; showed patterns of resilience; had reduced symptoms of anxiety and depression and an improved quality of life (Fonseca, Nazare, \& Canavarro, 2012). It would be useful to compare if having supportive, DDN trained staff can shorten the length of time it takes parents to adjust to the news and mitigate the impact that this has on their emotional, mental and family wellbeing as well as the potential long term effects of perinatal mental problems on both parents and children. 
In order to close the gap between evidence and practice, it is important to use findings from this study and any follow-on studies to lobby the development of policy to ensure that DDN training becomes part of mandatory training for relevant NHS staff and receives adequate funding to address the logistics of delivery of the training. This would need to also be complimented by policy and practice to ensure adequate provision of support for staff who deliver different news such as restorative supervision given the demands of DDN. This infrastructure would support large scale rolling out of the training to all staff and ensure consistent, safe and balanced practice and address the unmet need for training in DDN.

\section{Key Messages}

The DDN training has the potential to provide essential skills to HCPs who deliver DN to parents. Equipping HCPs with the necessary skills to effectively deliver DN may reduce the negative impact of the news on parents, families, and HCPs. If delivered well, there is potential to minimise the distress, anxiety, and depression associated with receiving different news. The improved mental wellbeing and adjustment of parents will also affect the mental health of their children which represents a key aspect of the prevention of mental ill health across the life course. 


\section{References}

Atkins, L., Francis, J., Islam, R., O'Connor, D., Patey, A., Ivers, N., . . Michie, S. (2017). A guide to using the Theoretical Domains Framework of behaviour change to investigate implementation problems. Implementation Science, 12. doi:10.1186/s13012-0170605-9

Cane, J., O'Connor, D., \& Michie, S. (2012). Validation of the theoretical domains framework for use in behaviour change and implementation research. Implementation Science, 7. doi:10.1186/1748-5908-7-37

Deater-Deckard, K. (2005). Parenting stress and children's development: Introduction to the special issue. Infant and Child Development, 14(2), 111-115. doi:10.1002/icd.383

Dent, K. M., \& Carey, J. C. (2006). Breaking difficult news in a newborn setting: Down syndrome as a paradigm. American Journal of Medical Genetics Part C-Seminars in Medical Genetics, 142C(3), 173-179. doi:10.1002/ajmg.c.30100

Drury, S. S., Scaramella, L., \& Zeanah, C. H. (2016). The Neurobiological Impact of Postpartum Maternal Depression: Prevention and Intervention Approaches. Child and Adolescent Psychiatric Clinics of North America, 25(2), 179-+. doi:10.1016/j.chc.2015.11.001

Fonseca, A., Nazare, B., \& Canavarro, M. C. (2012). Parental psychological distress and quality of life after a prenatal or postnatal diagnosis of congenital anomaly: A controlled comparison study with parents of healthy infants. Disability and Health Journal, 5(2), 67-74. doi:10.1016/j.dhjo.2011.11.001

Fonseca, A., Nazare, B., \& Canavarro, M. C. (2014). Parenting an infant with a congenital anomaly: An exploratory study on patterns of adjustment from diagnosis to six months post birth. Journal of Child Health Care, 18(2), 111-122. doi:10.1177/1367493512473856

Hoffman, C., Dunn, D. M., \& Njoroge, W. F. M. (2017). Impact of Postpartum Mental Illness Upon Infant Development. Current Psychiatry Reports, 19(12), 6. doi:10.1007/s11920017-0857-8

Lancaster, G. A., Dodd, S., \& Williamson, P. R. (2004). Design and analysis of pilot studies: recommendations for good practice. Journal of Evaluation in Clinical Practice, 10(2), 307-312. doi:10.1111/j..2002.384.doc.x

Luz, R., George, A., Spitz, E., \& Vieux, R. (2017). Breaking bad news in prenatal medicine: a literature review. Journal of Reproductive and Infant Psychology, 35(1), 14-31. doi:10.1080/02646838.2016.1253052

Michie, S., Atkins, L., \& West, R. (2014). The Behaviour Change Wheel: A Guide to Designing. Sutton: Silverback Publishing.

Morse, J. M. (2015). "Data Were Saturated ...". Qualitative Health Research, 25(5), 587-588. doi:10.1177/1049732315576699

Mugweni, E., Walker, M., Goodliffe, S., Jaswal, S., Lowenhoffe, C., \& Kendall, S. (2019). Improving the delivery of different news to families by healthcare professionals. In.

NCARDRS. (2018). Congenital anomaly statistics 2016. Retrieved from https://assets.publishing.service.gov.uk/government/uploads/system/uploads/attac hment data/file/751553/Congenital anomaly statistics 2016.pdf

PHE (2018). NHS Fetal Anomaly Screening Programme. Retrieved from https://assets.publishing.service.gov.uk/government/uploads/system/uploads/attac hment data/file/749742/NHS fetal anomaly screening programme handbook FI NAL1.2 18.10.18.pdf 
RCN. (2013). Breaking bad news: supporting parents when they are told of their child's diagnosis RCN guidance for nurses, midwives and health visitors. https://www.rcn.org.uk/professional-development/publications/pub-004471

Ritchie, J., \& Spencer, L. (1994). Qualitative data analysis for applied policy research. In A.Bryman \& R. G. Burgess (Eds.), Analyzing qualitative data (pp. 173-194.).

Sheets, K. M., Baty, B. J., Vazquez, J. C., Carey, J. C., \& Hobson, W. L. (2012). Breaking Difficult News in a Cross-cultural Setting: a Qualitative Study about Latina Mothers of Children with Down Syndrome. Journal of Genetic Counseling, 21(4), 582-590. doi:10.1007/s10897-011-9425-2

Skotko, B., \& Bedia, R. C. (2005). Postnatal support for mothers of children with Down syndrome. Mental Retardation, 43(3), 196-212. doi:10.1352/00476765(2005)43[196:psfmoc]2.0.co;2

Stein, A., Pearson, R. M., Goodman, S. H., Rapa, E., Rahman, A., McCallum, M., . . Pariante, C. M. (2014). Effects of perinatal mental disorders on the fetus and child. Lancet, 384(9956), 1800-1819.

Wen, D. J., Poh, J. S., Ni, S. N., Chong, Y. S., Chen, H., Kwek, K., . . Qiu, A. (2017). Influences of prenatal and postnatal maternal depression on amygdala volume and microstructure in young children. Translational Psychiatry, 7. doi:10.1038/tp.2017.74 


\section{List of Figures}

FIGURE 1: READY MNEMONIC

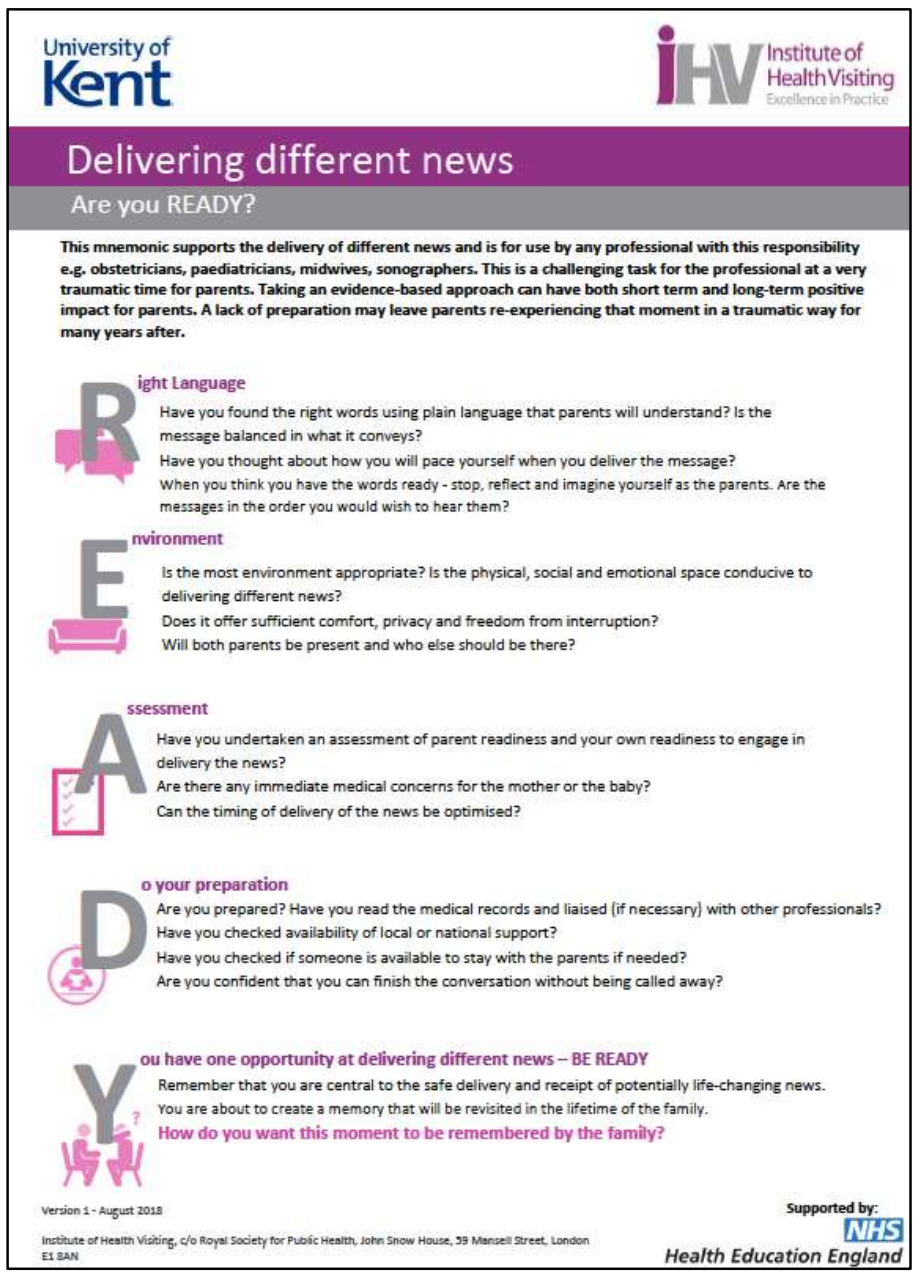

FIGURE 2: DESCRIPTION OF PROFESSIONALS WHO ATTENDED THE TRAINING

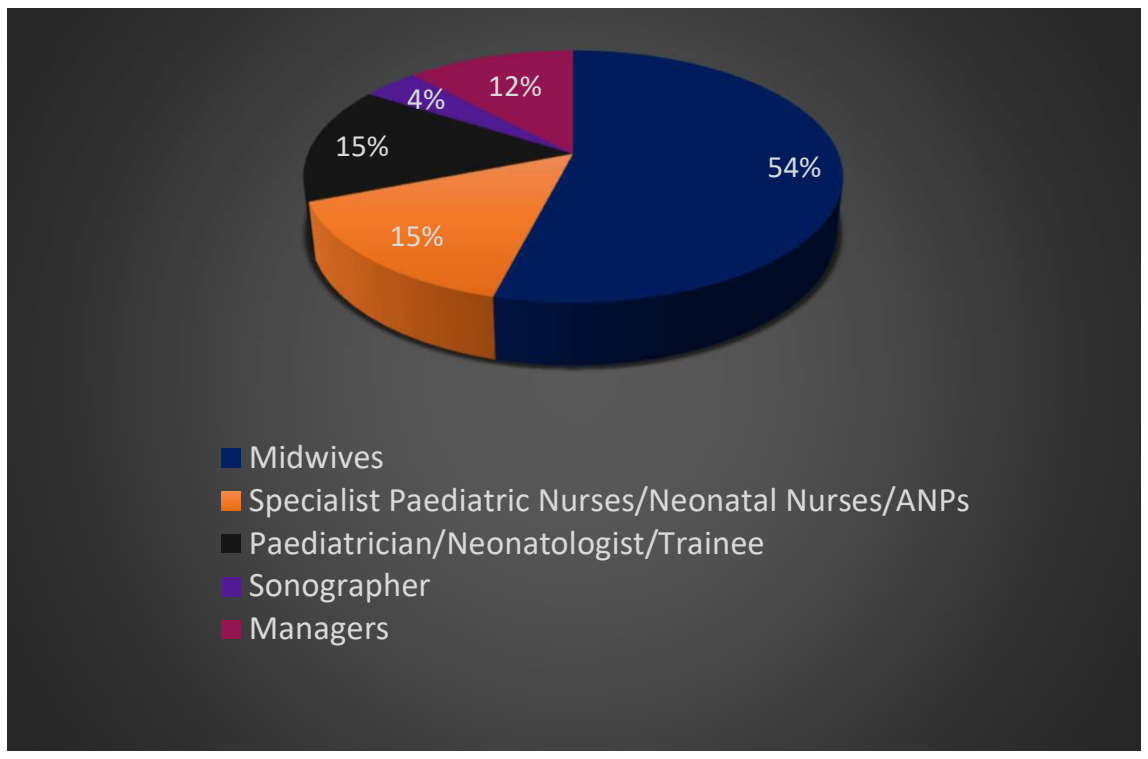


FIGURE 3: PREVIOUS TRAINING IN DDN

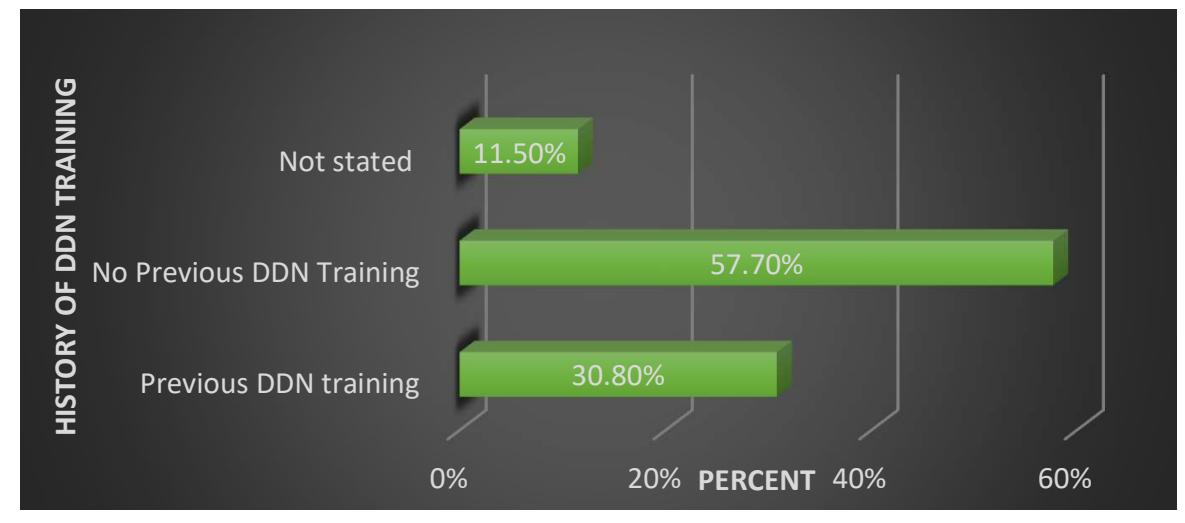

